\title{
Therapeutical doses of temozolomide do not impair the function of dendritic cells and $\mathrm{CDB}^{+} \mathrm{T}$ cells
}

\author{
$\mathrm{XUN} \mathrm{XU}^{1,2,3}$, FLORIAN STOCKHAMMER ${ }^{4,5}$, ANITA SCHMITT ${ }^{2,6}$, ROSAELY CASALEGNO-GARDUNO ${ }^{2}$, \\ ANNE ENDERS $^{7}$, JIJU MANI $^{2,6}$, CARL FRIEDRICH CLASSEN $^{7}$, MICHAEL LINNEBACHER $^{8}$, \\ MATHIAS FREUND ${ }^{2}$ and MICHAEL SCHMITT ${ }^{2,6}$ \\ ${ }^{1}$ Department of Immunology, Jiangsu University, Zhenjiang 212013, P.R. China; ${ }^{2}$ Department of Internal Medicine III, \\ University of Rostock, D-18057 Rostock; ${ }^{3}$ Center for Biomaterial Development and Berlin-Brandenburg Center for \\ Regenerative Therapies, Institute of Polymer Research, Helmholtz-Zentrum Geesthacht, D-14513 Teltow; ${ }^{4}$ Department of \\ Neurosurgery, University of Rostock, D-18057 Rostock; ${ }^{5}$ Department of Neurosurgery, University of Göttingen, \\ D-37073 Göttingen; ${ }^{6}$ Department of Medicine V, University of Heidelberg, D-69120 Heidelberg; \\ Departments of ${ }^{7}$ Pediatrics and ${ }^{8}$ Surgery, University of Rostock, D-18057 Rostock, Germany
}

Received July 16, 2011; Accepted September 5, 2011

DOI: $10.3892 /$ ijo.2011.1269

\begin{abstract}
The median survival of patients with glioblastoma multiforme (GBM) remains poor. Innovative immunotherapies with dendritic cell (DC) vaccination might be combined with standard temozolomide (TMZ) treatment. Here, we evaluated the influence of TMZ on the phenotype and function of DCs and $\mathrm{CD}^{+} \mathrm{T}$ cells. DCs were generated from the peripheral blood of healthy volunteers (HVs) and GBM patients. DCs were analyzed by light microscopy and flow cytometry. Phagocytic activity was tested by FITC-dextran engulfment. Mixed lymphocyte peptide cultures were followed by enzyme-linked immunospot (ELISPOT) and flow cytometry assays. TMZ was added to DC and T cell cultures at concentrations up to $500 \mu \mathrm{M}$. Mature DCs were generated from HVs and GBM patients. Cells displayed a typical DC morphology and a mature DC phenotype. Expression of CD209 was even higher in DCs generated from patients under therapy than from HVs (75.2 vs. 51.1\%). In contrast, CD40 (1.1 vs. $13.5 \%$ ) and BDCA4 (26.5 vs. 52.9\%) were lower expressed in GBM patients at time of diagnosis. Immature DCs showed high phagocytic activity. Addition of TMZ at concentrations up to $50 \mu \mathrm{M}$ did neither impair the phenotype nor the function of DCs. In ELISPOT and flow cytometry assays, no impairment of $\mathrm{CD} 8^{+} \mathrm{T}$ cell responses to viral antigens could be observed. Taken together, TMZ does not impair the function of either DCs or the $\mathrm{CD} 8^{+} \mathrm{T}$ cells.
\end{abstract}

\section{Introduction}

The outcome of patients with glioblastoma multiforme (GBM), the most common and the most aggressive malignant glioma

Correspondence to: Dr Michael Schmitt, Department of Medicine V, University of Heidelberg, D-69120 Heidelberg, Germany

E-mail: michael.schmitt@med.uni-heidelberg.de

Key words: dendritic cells, glioblastoma, temozolomide, $\mathrm{T}$ cells
(WHO grade IV), still remains poor with a median survival time of 14 months after first diagnosis (1). Standard therapy is maximum surgical cytoreduction without causing new neurological deficit followed by a total dose of 60 Gray-fractionated focal irradiation with concomitant temozolomide (TMZ) chemotherapy $(2,3)$. TMZ is a prodrug which is metabolized to the more active metabolite 5-triazeno imidazole carboxamide acting as an alkylating agent. Standard first line therapy in glioblastomas consists of a continuous dosing of $75 \mathrm{mg} / \mathrm{m}^{2} \mathrm{TMZ}$ concomitant to radiotherapy, followed by 6 adjuvant cycles of $150-200 \mathrm{mg} / \mathrm{m}^{2}$ TMZ from day 1 to 5 of a 28 -day cycle. TMZ serum levels of the patients under TMZ therapy can reach up to $50 \mu \mathrm{M}$ during therapy (4). Innovative immunotherapeutic approaches might combine dendritic cell (DC) vaccination with standard TMZ therapy.

DCs are professional antigen-presenting cells with the ability to present peptide epitopes both on classes I and II molecules of the major histocompatibility complex (MHC). Recently, several groups (5-9) demonstrated clinical and immunological responses of glioblastoma patients after intradermal injection of autologous DCs loaded with tumor lysate in the vicinity of lymph nodes, despite several hints indicating that glioblastomas have a strong immunosuppressive capacity.

In this study, we investigated the generation of monocyte-derived autologous DCs from the peripheral blood of glioblastoma patients in comparison to healthy volunteers (HVs) with regard to phenotype, morphology and function in the presence of therapeutical levels of TMZ. Furthermore, the effect of TMZ on $\mathrm{CD}^{+} \mathrm{T}$ cells was investigated.

\section{Materials and methods}

Source of TMZ. TMZ was obtained from Sigma-Aldrich (Munich, Germany). It was dissolved in dimethyl sulfoxide (DMSO) (Merck, Darmstadt, Germany) at a final concentration of $0.5 \%$ DMSO, a non-toxic concentration. DMSO control experiments were performed in order to exclude toxic 
effects of DMSO following data earlier published by Pera et al (10). TMZ concentrations ranged from 1 to $100 \mu \mathrm{g} / \mathrm{ml}$ $(5-500 \mu \mathrm{M})$.

Peripheral blood samples from HVs and GBM patients. Peripheral blood mononuclear cells (PBMCs) were isolated with Biocoll Separation Solution (Biochrom, Berlin, Germany) density gradient centrifugation of ethylenediaminetetraacetic acid (EDTA; Delta-Pharma, Pfullingen, Germany) anticoagulated blood. Peripheral blood samples from 5 patients with newly diagnosed GBM and 6 patients under therapy were taken. Table I displays the characteristics of these GBM patients. Control samples were prepared from $12 \mathrm{HVs}$. Informed consent was given by all patients and HVs, the study was approved by the Rostock University ethics committee (vote no. A2009 34). The viability of PBMCs obtained was always $>95 \%$, as determined by trypan blue staining (Trypan Blue Solution $0.4 \%$, Sigma-Aldrich). The viable cells were quantified in a Neubauer chamber (Zeiss, Oberkochen, Germany). For DC generation, Biocoll separated PBMCs were cryopreserved in RPMI-1640 (Biochrom AG, Berlin, Germany) containing 20\% human AB serum (Blood bank, University of Rostock, Germany) and 10\% DMSO and immediately stored at $-80^{\circ} \mathrm{C}$.

Generation of DCs and TMZ treatment. Generation of DCs from peripheral blood cells was performed as described previously (11-14). Briefly, PBMCs were seeded $\left(2.5 \times 10^{6}\right.$ cells/ $\mathrm{ml} /$ well) into 6-well plates (Becton-Dickinson, Heidelberg, Germany) in RPMI-1640 (Biochrom AG) supplemented with $2 \%$ human $\mathrm{AB}$ serum, $1 \% \mathrm{~L}$-glutamine, $1 \%$ penicillin and streptomycin. After $2 \mathrm{~h}$ of incubation at $37^{\circ} \mathrm{C}$, non-adherent cells were removed. The remaining adherent cells (including $70 \%$ $\mathrm{CD} 14^{+}$cells) from HVs or patients, respectively, were cultured in RPMI-1640 supplemented with $100 \mathrm{ng} / \mathrm{ml}$ human GM-CSF, $1000 \mathrm{IU} / \mathrm{ml}$ IL-4 (Miltenyi-Biotec, Bergisch Gladbach, Germany), and $10 \%$ human AB serum. For maturation of the cells, medium change was performed on day +6 . The new medium contained GM-CSF and IL-4 in the concentrations mentioned above in addition of $50 \mathrm{ng} / \mathrm{ml}$ TNF- $\alpha$ (MiltenyiBiotec). Cell differentiation was monitored by inverse light microscopy. TMZ was added to DC culture medium on day 0 of DC generation. TMZ in different concentrations $(0,50$, 100,250 and $500 \mu \mathrm{M}$ ) was added to the DC culture medium after plastic adherence. Afterwards, DCs were continuously cultured for 6 days.

Viability staining and morphological studies of DCs. The viability of the DCs harvested after 8 days of culture was assessed by trypan blue staining and the morphology of the cells was analyzed by light microscopy. Furthermore, cells were stained with $1 \mathrm{nM} / \mathrm{ml}$ Calcein AM (Invitrogen $\mathrm{GmbH}$, Karlsruhe, Germany) in order to quantify viable cells. In each experiment untreated cells served as a control. Cells treated with $70 \%$ methanol prior to staining served as dead control. Analysis was performed on a fluorescence multi-well plate reader (Tecan Infinite M200, Crailsheim, Germany) at an excitation wavelength of $485 \mathrm{~nm}$ (emission $535 \mathrm{~nm}$ ). DC suspensions were spun on slides and stained with May-Gruenwald-Giemsa solution (Merck, Darmstadt, Germany).
Table I. Clinical characteristics of the GBM patients included in this study.

\begin{tabular}{ccclc}
\hline No. & Sex & Age & \multicolumn{1}{c}{ Status } & TMZ therapy \\
\hline 1 & M & 76 & At diagnosis & No \\
2 & M & 68 & At diagnosis & No \\
3 & F & 77 & At diagnosis & No \\
4 & F & 71 & At diagnosis & No \\
5 & M & 72 & At diagnosis & No \\
6 & F & 70 & At relapse & Yes \\
7 & F & 54 & At relapse & Yes \\
8 & M & 72 & In remission & Yes \\
9 & F & 60 & At relapse & Yes \\
10 & F & 63 & At relapse & Yes \\
11 & M & 70 & In remission & Yes \\
\hline
\end{tabular}

TMZ, temozolomide; M, male; F, female.

Surface marker analysis. The expression of cell surface molecules on the DCs was analyzed by flow cytometry after 8 days of culture. Harvested cells were washed in fluorescenceactivated cell sorting (FACS) medium [phosphate-buffered saline (PBS) containing 1\% bovine serum albumin (BSA)] and stained at $4^{\circ} \mathrm{C}$ for $20 \mathrm{~min}$ with anti-human CD14, CD40,CD83, CD80, CD86, CD205, CD209, HLA-DR, BDCA1, BDCA4, (Becton-Dickinson, Heidelberg, Germany) and CD11c (ImmunoTools, Friesoythe, Germany) antibodies directly conjugated with FITC, PE, PerCP, APC, PE-Cy7, APC-Cy7, Alexa 647 or V450. Thereafter, cells were washed three times with 1X PBS and analyzed in a flow cytometer (FACSAria ${ }^{\mathrm{TM}}$, BD Biosciences, Heidelberg, Germany) using FACSDiva software (FACSDiva V6.1.2, BD Biosciences, Heidelberg, Germany). Negative controls consisted of DCs stained with the appropriate isotype controls. The number of positive cells was assessed in a gate characterized by a large size and a dense granularity. Results were visualized in the dot plot and histogram format.

Treated DCs were incubated with mouse anti-human monoclonal antibodies: APC-conjugated CD83 $(0.2 \mu \mathrm{g}$ clone HB15) and PE-conjugated CD86 (0.2 $\mu \mathrm{g}$ clone M95, both Miltenyi Biotec, Bergisch-Gladbach, Germany). The number of DCs was quantified by addition of fluorescent microsphere beads (Polysciences, Eppelheim, Germany). For each sample 7500 events were measured by flow cytometric analysis. Data are given as frequencies of DCs compared to untreated controls (normalized as 1). Data analysis was performed on a FACSCalibur using CellQuest software (BD Biosciences, Heidelberg, Germany).

Phagocytic activity and detection of IL-12p70 in enzymelinked immunosorbent assays (ELISA). Both day +6 immature DCs and day +8 mature DCs were collected and subsequently exposed to FITC-dextran $(1 \mathrm{mg} / \mathrm{ml})$ for $30 \mathrm{~min}$ at $4^{\circ} \mathrm{C}$ or at $37^{\circ} \mathrm{C}$, and examined by flow cytometry to measure non-specific and specific uptake, respectively. The IL-12p70 enzyme-linked immunosorbent assay (ELISA) kit was used according to the manufacturer's instructions to analyze the production of 
A
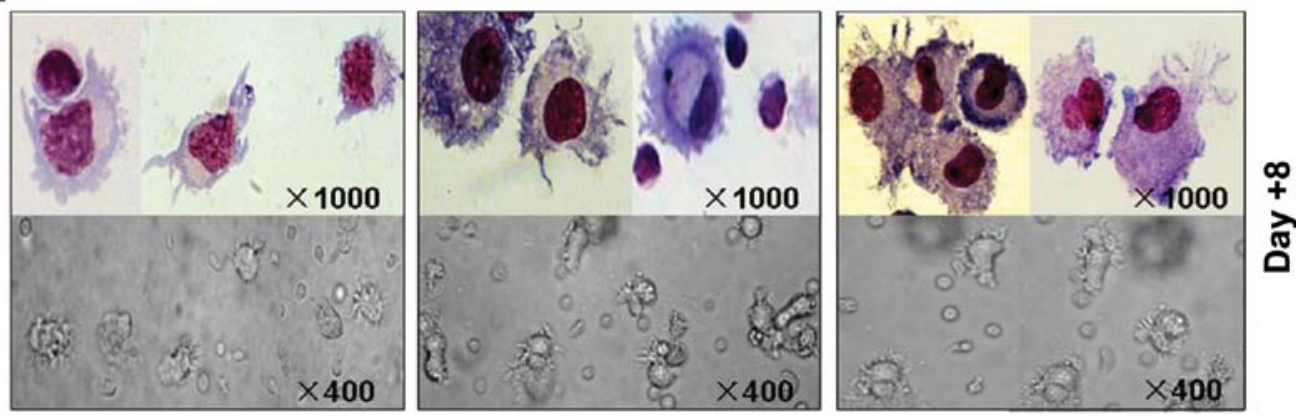

B

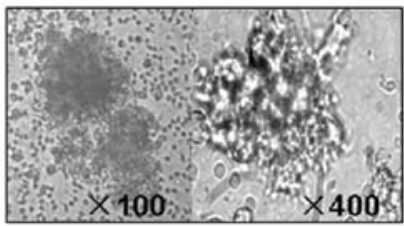

HV-DCs

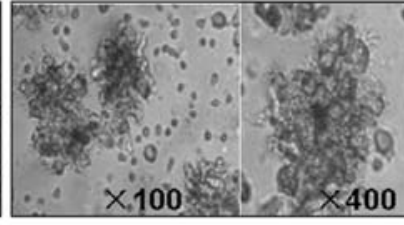

GBM-DCs

(At diagnosis)

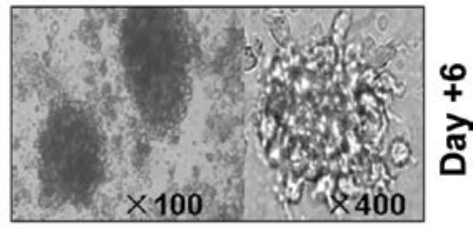

GBM-DCs

(Under therapy)

Figure 1. Morphology of HV-DCs and GBM-DCs. DCs generated from PBMCs of HVs (left), GBM patients at time of diagnosis (middle) and under therapy (right) were harvested on (A) day +6 and (B) day +8 . The morphology of the cells was analyzed by light microscopy. Magnifications are indicated.

IL-12p70 (R\&D Systems, Wiesbaden, Germany) by DCs. Each condition was measured in duplicate. The supernatants for ELISA were kept at $-20^{\circ} \mathrm{C}$ prior to analysis.

Mixed lymphocyte peptide culture (MLPC). MLPC was performed as described earlier $(15,16)$. Briefly, after isolation of $\mathrm{CD}^{+} \mathrm{T}$ lymphocytes from the Cytomegalovirus (CMV) pp65tetramer ${ }^{+}$healthy volunteer, CD8- APCs were irradiated with 30 Gy and pulsed with the peptide of CMVpp65 (Sequence: NLVPMVATV) or receptor for hyaluronan-mediated motility derived peptide R3 [RHAMM-R3 (sequence: ILSLELMKL)] at a concentration of $20 \mu \mathrm{g} / \mathrm{ml}$ for $2 \mathrm{~h}$. After co-incubation with $\mathrm{CD} 8^{+} \mathrm{T}$ lymphocytes in the presence or absence of $\mathrm{TMZ}$ overnight at $37^{\circ} \mathrm{C}, 5 \% \mathrm{CO}_{2}$ and $99 \%$ humidity, the MLPC was supplemented with $10 \mathrm{IU} / \mathrm{ml} \mathrm{IL-2}$ and $20 \mathrm{ng} / \mathrm{ml} \mathrm{IL-7.} \mathrm{After} \mathrm{a}$ total of 8 days of culture, cells were harvested and evaluated for their IFN- $\gamma$ secretion by an ELISPOT assay employing T2 cells pulsed with CMVpp65 or RHAMM-R3 peptide. Proliferation of $\mathrm{CMVpp} 65$-specific $\mathrm{CD} 8^{+} \mathrm{T}$ cells was determined after one round of MLPC by staining with CD8-FITC antibody and HLA-A2/CMVpp65 peptide tetramer PE.

Enzyme-linked immunospot assay (ELISPOT). IFN- $\gamma$ ELISPOT assays were performed as previously described (17). Presensitized CD8 ${ }^{+} \mathrm{T}$ lymphocytes $\left(1 \times 10^{4}\right)$ and $4 \times 10^{4}$ target cells (CMVpp65 or RHAMM-R3 peptide pulsed T2 cells) were added to each well. The spots were evaluated by the use of an ELISPOT reader (CTL, Reutlingen, Germany). The results are presented as an average of duplicate cultures.

Statistical analysis. Statistical analyses were calculated using Statistical Product and Services Solutions (SPSS) package (SPSS Version 11.5 for Windows, SPSS Science, Chicago, USA). The significance of the difference between means was determined by the analysis of one-way variance (ANOVA), and the differences were considered statistically significant at $\mathrm{P}<0.05$.

\section{Results}

Typical morphology of both HV-DCs and GBM-DCs. After eight days of culture, floating and semi-adherent cells generated from monocytes of GBM patients (GBM-DCs) and of HVs (HV-DCs) displayed a typical DC morphology with cytoplasmic veils (Fig. 1A). Both DC precursors showed typical dendrites and colony formation in DC medium by phase contrast microscopy on day +6 (Fig. 1B). The viability of the DC preparations was always $>95 \%$ as determined by trypan blue staining (data not shown).

Phenotype of DCs generated from PBMCs of HVs and GBM patients in vitro. On day +8 , both HV-DC and GBM-DC preparations showed the phenotypic characteristics of monocyte-derived DCs, i.e. high level expression of MHC class II (HLA-DR) and CD86 and none or weak CD14. Flow cytometric analysis was performed for $12 \mathrm{HV}$-DC and for 11 GBM-DC preparations as shown in Fig. 2A. No significant differences between HV-DCs and GBM-DCs of patients at time of diagnosis $(n=5)$ or under therapy $(n=6)$ were observed concerning the expression of HLA-DR (median $97.7 \%$ in healthy volunteers versus $99.0 \%$ in GBM patients at time of diagnosis and 99.0\% in patients under therapy) and $\mathrm{CD} 83^{+} \mathrm{CD} 86^{+}$cells (47.5 vs. 48.4 and $36.9 \%$ ). This high percentage demonstrates effective maturation of the DCs. Expression of CD209 (75.2 vs. $51.1 \%, \mathrm{P}<0.05)$ was even higher in DCs generated from GBM patients under therapy than from HVs. However, expression of CD40 (1.1 vs. $13.1 \%, \mathrm{P}<0.05)$ and BDCA4 (25.2 vs. $56.5 \%$, $\mathrm{P}<0.05$ ) was lower in patients at time of diagnosis (Fig. 2B). 
A


CD205

BDCA4

BDCA1

CD14

CD11c

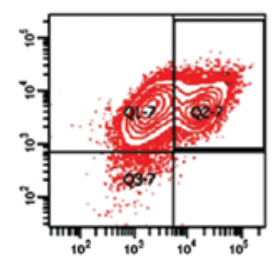

GBM-DCs
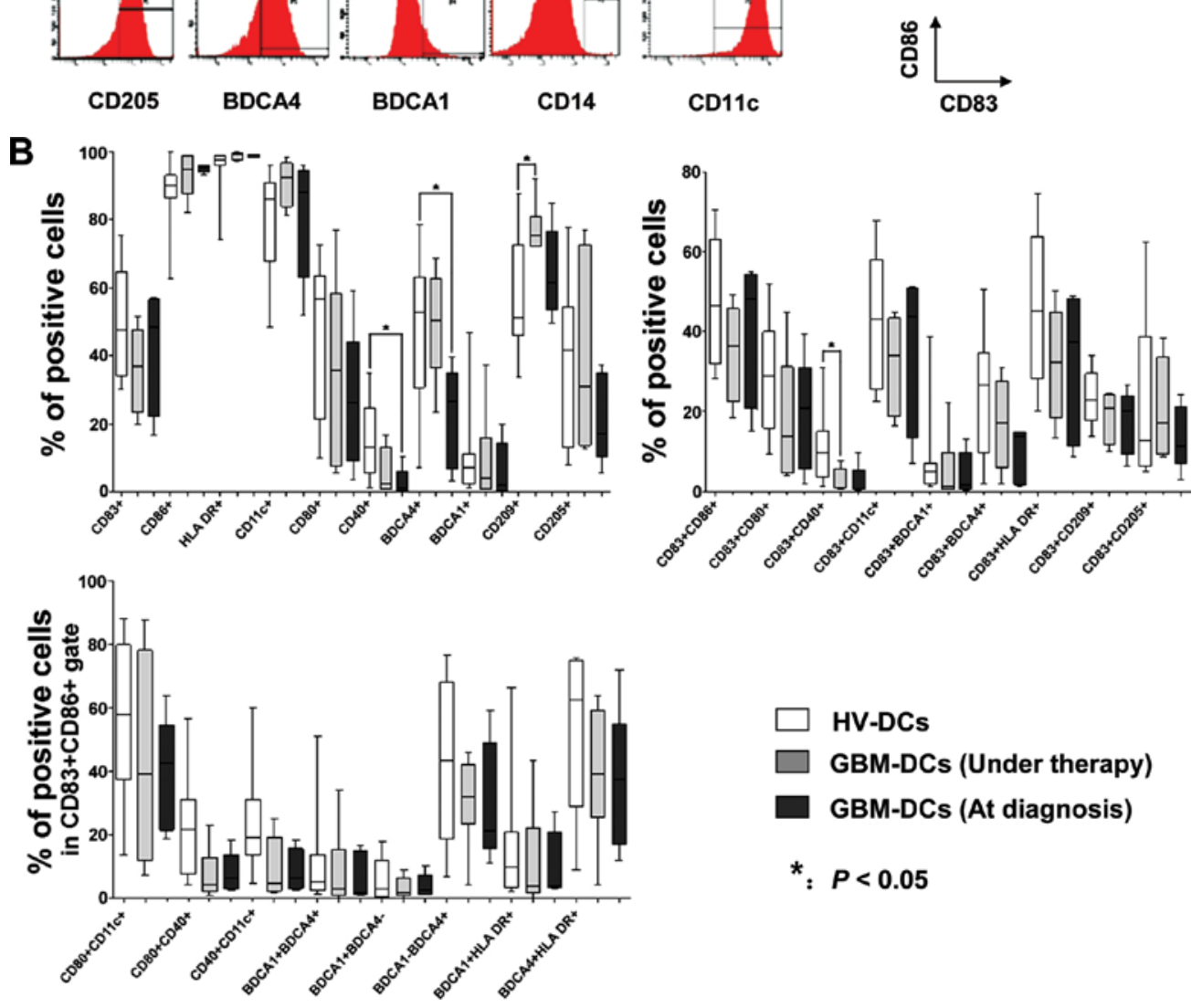

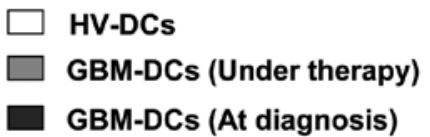

$*: P<0.05$

Figure 2. Immunophenotype analyses of HV-DCs in comparison to GBM-DCs. Mature DCs generated from the peripheral blood of 12 HVs and 11 GBM patients were collected on day +8 and subjected to flow cytometrical analysis. (A) Histograms show the expression of the surface markers on mature DCs of a representative HV and a GBM patient. (B) Box plots summarize the distribution of the different surface markers on mature HV-DCs (n=12) and GBM-DCs from newly diagnosed patients $(\mathrm{n}=5)$ and patients under therapy $(\mathrm{n}=6)$ including minimum, maximum and median values. For $\mathrm{CD} 83^{+} \mathrm{CD} 86^{+} \mathrm{DCs}$ the coexpression of CD11c, CD40, CD80, CD205, CD209, HLA-DR, BDCA1 and BDCA4 was further analysed (lower part of panel B). "P<0.05.

Phagocytic function of $H V-D C s$ and GBM-DCs. Both day +6 immature DCs and day $+8 \mathrm{TNF}-\alpha$-stimulated DCs were collected and subsequently exposed to FITC-conjugated dextran $(1 \mathrm{mg} / \mathrm{ml})$ for $30 \mathrm{~min}$ at $4^{\circ} \mathrm{C}$ or at $37^{\circ} \mathrm{C}$ and examined by flow cytometry to measure non-specific and specific uptake, respectively. A high level of FITC-dextran uptake was shown in the histograms of immature DCs generated from both HVs and patients. TNF- $\alpha$-induced maturation of DCs was char- acterized by a decrease of endocytosis. Similar results were obtained in five experiments. One representative experiment is displayed in Fig. 3. DCs generated from an HV displayed $79.8 \%$ endocytosis on day +6 vs. $18.4 \%$ endocytosis on day +8 and DCs from a GBM patient displayed 91.3 vs. $4.5 \%$ (Fig. 3).

The impact of high dose TMZ on the frequency and IL-12 secretion of DC. We generated HV-DCs with the conventional 


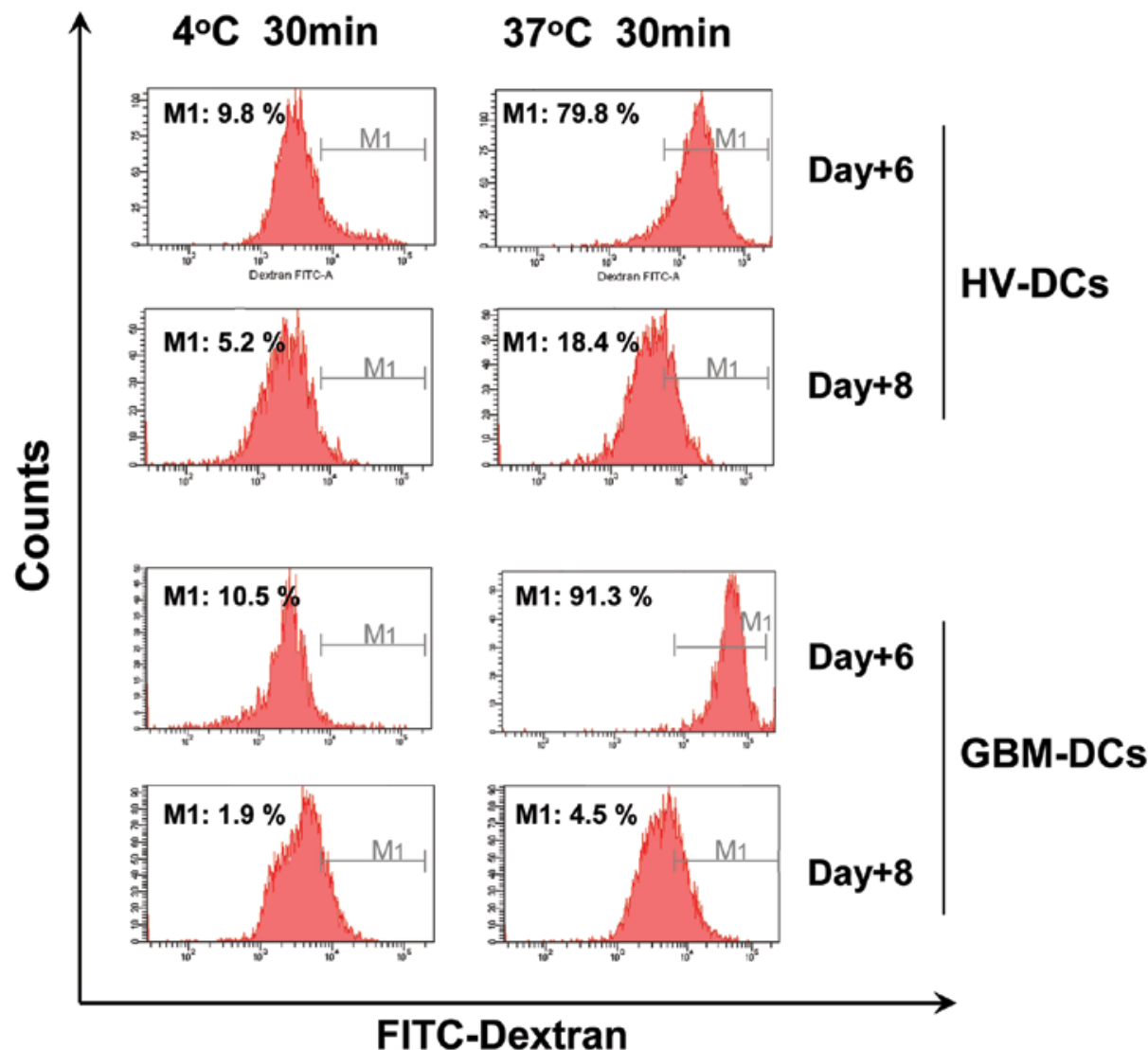

Figure 3. Functional analysis of DCs by FITC-dextran endocytosis. Both day +6 immature DCs and day +8 mature DCs were collected and subsequently exposed to FITC-dextran and examined by flow cytometry. The histograms show immature and mature DCs of an exemplary healthy volunteer (upper part) and a GBM patient (lower part) exposed to FITC-dextran for 30 min at $4^{\circ} \mathrm{C}$ (left row) vs. $37^{\circ} \mathrm{C}$ (right row).


Figure 4. Effect of TMZ on the generation of DCs. TMZ was added to DC culture medium on day 0 of DC generation at a final concentration of $0,50,100,250$ or $500 \mu \mathrm{M}$, respectively. On day +8 mature DCs were collected and immunophenotyped by flow cytometry. The dot plots show the exact number of CD83 ${ }^{+} \mathrm{CD} 86^{+}$ DCs measured in parallel to 7,500 fluorescent microsphere beads added to all samples for normalization. CD83 ${ }^{+} \mathrm{CD} 86^{+} \mathrm{DC}$ generation with TMZ addition according to the final concentration of TMZ is indicated. Untreated cells were used as control and set 1 . The asterisks indicate that the reduction of the cell number was significant at a level of $\mathrm{P}<0.05$. 
A



B

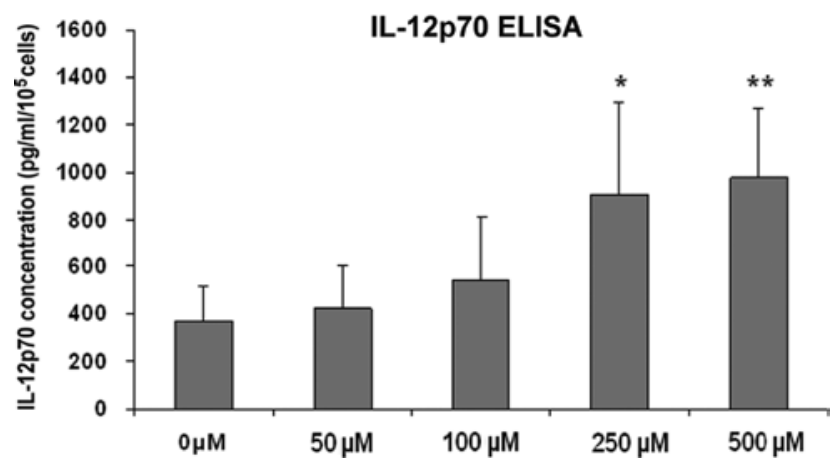

Figure 5. Quantitative analysis of DC viability and IL-12p70 secretion under TMZ-treatment. (A) The histogram shows the viability of TMZ (5-500 $\mu \mathrm{M})$ treated DCs harvested on day +8 . Cells were stained with calcein AM. Data are given as $\mathrm{x}$-fold number of living DCs compared to untreated control DCs (live control). (B) On day +8 the supernatants from DCs, which were exposed to different concentration of $\operatorname{TMZ}(0,50,100,250$ or $500 \mu \mathrm{M})$, were collected. The IL-12p70 concentration was analyzed in duplicate using ELISA $(n=5)$. ${ }^{*} \mathrm{P}<0.05,{ }^{* *} \mathrm{P}<0.01$.

cytokine cocktail for 6 days in the presence of different concentrations of TMZ ranging from 0 to $500 \mu \mathrm{M}$. Subsequently, the immature DCs were matured with TNF- $\alpha$. Under these conditions, TMZ inhibited the DC generation in a dose-dependent manner. By increasing the TMZ concentration from 5, 50, 100, 250 to $500 \mu \mathrm{M}$, the frequencies of $\mathrm{CD}^{2} 3^{+} \mathrm{CD} 86^{+} \mathrm{DCs}$ generated from HVs were 1.07-, 0.96-, 0.69-, 0.64- and 0.45-fold compared to the group without TMZ (Fig. 4). Cell numbers determined by viability staining were $1.03-, 0.89-, 0.86-, 0.92-$, 0.74-fold, respectively, normalized to untreated living control cells (Fig. 5A). Although the frequencies of $\mathrm{CD} 83^{+} \mathrm{CD} 86^{+}$cells were increased in the $5 \mu \mathrm{M}$ TMZ group and decreased in the $50 \mu \mathrm{M}$ group this did not reach statistical significance. These results at least indicate that therapeutic levels of TMZ of up to $50 \mu \mathrm{M}$ do not impair the generation of DCs from HVs in vitro (Fig. 4). However, TMZ levels higher than $250 \mu \mathrm{M}$ did reduce significantly $(\mathrm{P}<0.05)$ the number of viable cells (Fig. 5A).

IL-12p70 secretion of mature DCs from HVs was assessed by ELISA. Bioactive IL-12p70 secretion was achieved in the presence of TNF- $\alpha$ in the DC medium between day +6 and day +8 of generation of mature DCs and reached 1,066 pg/ $\mathrm{ml}$ (median, $\mathrm{n}=5$ ). Finally, we investigated whether DCs retain the ability to secrete bioactive IL-12p70 during the maturation step upon exposure to different concentrations of TMZ (50, $100,250,500 \mu \mathrm{M})$ during 6 days of DC generation. On day +6 , the immature HV-DCs were washed twice and transferred to a new culture plate to assess the IL-12 production. After exposure to TNF- $\alpha$ for $48 \mathrm{~h}$, we observed a median secretion of bioactive IL-12p70 with respectively $1,233 \mathrm{pg} / \mathrm{ml}$ in the $50-\mu \mathrm{M}$ TMZ group, $1,199 \mathrm{pg} / \mathrm{ml}$ in the $100-\mu \mathrm{M}$ TMZ group, $1,160 \mathrm{pg} / \mathrm{ml}$ in the $250-\mu \mathrm{M} \mathrm{TMZ}$ group and $1,051 \mathrm{pg} / \mathrm{ml}$ in the $500-\mu \mathrm{M}$ TMZ group. The secretion of IL-12p70 was higher in the 50-, 100- and 250- $\mu \mathrm{M}$ TMZ groups and decreased with higher levels of TMZ (data not shown).

As addition of TMZ to DC cultures resulted in a decrease of the number of cells (Fig. 5A), we decided to normalize the secretion of IL-12 to a number of $10^{5}$ living cells. Fig. 5B gives the results of the normalized IL-12 production. At concentrations of $250(\mathrm{P}<0.05)$ and $500 \mu \mathrm{M}$ TMZ significantly $(\mathrm{P}<0.01)$ higher IL-12 levels were observed per cell as compared to lower concentrations.

Impact of TMZ on $T$ cell function. $\mathrm{CD}^{+} \mathrm{T}$ lymphocytes from CMVpp65-tetramer ${ }^{+} \mathrm{HVs}$ were subjected to one round of stimulation with irradiated autologous CD8- cells pulsed with CMVpp65 peptide or control peptide (RHAMM-R3) in the absence or presence of different concentrations of TMZ (varying from 5 to $500 \mu \mathrm{M}$ ). Flow cytometric analyses were performed with anti-CD8 FITC/CD3 PerCP/CD14 APC/CD19 APC and CMV tetrameric complexes conjugated with PE to evaluate the promotion or inhibition of proliferation of relevant antigen specific $\mathrm{CD}^{+} \mathrm{T}$ lymphocytes by TMZ. In all samples, high doses $(500 \mu \mathrm{M})$ of TMZ reduced the T cell proliferation over 8 days, however, lower concentrations of TMZ (5-50 $\mu \mathrm{M})$ did not impair the proliferation of relevant antigen-specific $\mathrm{CD}^{+} \mathrm{T}$ cells. The frequency of $\mathrm{CD}^{+} \mathrm{T}$ cells within the $\mathrm{CD} 3^{+}$ lymphocyte gate specifically recognizing the CMV peptide were $80.7,81.9,81.5,51.1 \%$ with increasing TMZ concentrations $(0,5,50,500 \mu \mathrm{M})$, respectively (Fig. 6 upper panel).

To further evaluate the influence of TMZ on the function of $\mathrm{CD}^{+} \mathrm{T}$ cells from HVs, ELISPOT assays were performed to assess the CMVpp65-specific production of IFN- $\gamma$. TMZ did not significantly change the frequency of detectable spots with increasing concentrations up to $50 \mu \mathrm{M}$. However, with $500 \mu \mathrm{M} \mathrm{TMZ}$, the frequency of IFN- $\gamma$ spots was decreased when compared with the TMZ-untreated group and the RHAMM-R3-pulsed control group (Fig. 6 lower panel; one representative experiment of three is shown).

\section{Discussion}

Treatment of patients with glioblastoma multiforme (GBM) remains a major challenge for the oncologist.Despite multimodal therapy including surgery, radiotherapy and chemotherapy with temozolomide (TMZ), $<10 \%$ of the patients survive $>5$ years after diagnosis of the disease (3). Resistance to chemotherapy and serious toxicity of TMZ remain the major problems in the treatment of GBM patients with TMZ (18). Therefore, there is a fervent need for novel, potentially synergistic therapies for the disease.

Immunotherapy constitutes an alternative tumor-specific approach. For the initiation of tumor-specific immune responses, the expression of immunogenic tumor-associated antigens (TAA) is favorable. Examples of glioma-associated antigens capable of initiating $\mathrm{T}$ cell responses are HER2, gp100, MAGE-1, EphA2 and SOX11 (19-22). 
TMZ concentration

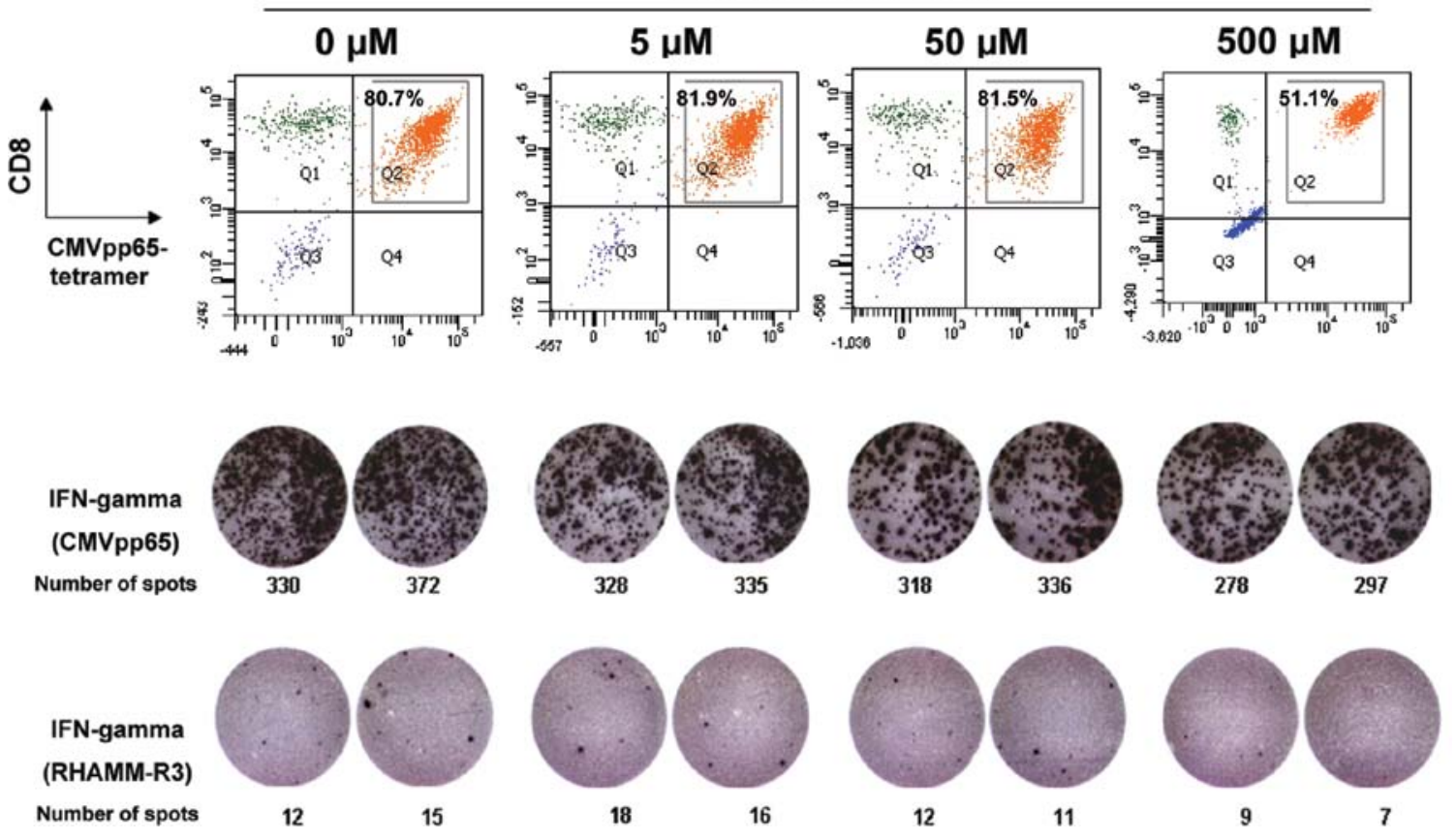

Figure 6. Effect of TMZ on antigen-specific T cell activation and proliferation. CD8 ${ }^{+} \mathrm{T}$ lymphocytes from HLA-A2 ${ }^{+} \mathrm{CMVpp} 65$-seropositive healthy donors were stimulated with irradiated and CMVpp65 peptide-loaded CD8- autologous cells in the presence or absence of TMZ (0,5, 50 and $500 \mu \mathrm{M})$. After a total of 8 days of culture, cells were harvested and evaluated for the frequency of CMV-specific CD8 ${ }^{+} \mathrm{T}$ cells (upper panel). Additionally, their potential to secrete IFN- $\gamma$ was measured by ELISPOT employing T2 cells pulsed with CMVpp65 (upper panel) or RHAMM-R3 control peptide (lower panel). Displayed are the representative results from one of three consecutive experiments.

Furthermore, Tang et al have shown that GBM patients have circulating tumor-specific $\mathrm{CD}^{+} \mathrm{T}$ cells concluding that GBM is an immunogenic tumor (23). These data support the development of an active specific immunotherapeutic approach for GBM patients.

Active immunotherapy with dendritic cells (DCs) as the most professional antigen-presenting cells (APCs) for the stimulation of $\mathrm{T}$ lymphocytes recognizing and eliminating tumor cells has been developed in recent years. Our group reported earlier that autologous DCs generated from leukemic blasts of patients with acute myeloid leukemia induced an enhanced specific cytotoxic $T$ cell response in these patients (11-13).

In spite of the location of the GBM in the so-called immuno-privileged environment of the central nervous system, promising clinical results of autologous DC-based tumor vaccination following surgery have been described (24-29). The group around van Gool $(5,7,8,30)$ demonstrated that autologous DC vaccination can induce long-term survival in GBM patients. Liau and co-workers (9) reported systemic anti-tumor $\mathrm{T}$ cell responses, an increased number of tumor infiltrating lymphocytes and a clinical response. Yu et al (28) observed cytotoxic T cell responses in $60 \%$ of glioma patients vaccinated with tumor lysate-loaded DCs, which was higher than the $40 \%$ seen with eluted peptides by the same group (24). In another phase I/II trial, one partial response and three minor responses were seen in 24 recurrent malignant glioma patients treated with intradermal injections of tumor lysateloaded DCs (31).

Based on these positive clinical findings we wondered: a) whether there are any differences in morphology, phenotype and function of autologous DCs generated from GBM patients at different clinical stages when compared to DCs generated from HVs, and b) whether therapy with TMZ has an influence on the generation of DCs and antigen-specific $\mathrm{CD} 8^{+} \mathrm{T}$ cells.

Accordingly, we generated DCs from both GBM patients (GBM-DCs) and healthy volunteers (HV-DCs). All DCs presented typical cytoplasmic veils (Fig. 1). Altogether, all DC preparations showed a similar phenotype (Fig. 2). However, a lower expression of CD40 on DCs generated from GBM patients at time of diagnosis and in the treatment course could generally be observed. Additionally, expression of BDCA4 on DCs generated from GBM patients at time of diagnosis was as high as on HV-DCs, but lower on DCs generated from GBM patients in the course of treatment (Fig. 2B). CD40 is a costimulatory protein required for the activation of APCs. Down-regulation of CD40 inhibits the proper activation of APCs. This has been suggested to be a mechanism of tumor escape from immune surveillance $(32,33)$. Interestingly, the expression of CD209 was higher on DCs generated at the time of diagnosis when compared with GBM-DCs in the course of treatment. CD209 (DC-SIGN; dendritic cell-specific intercellular adhesion molecule-3-grabbing non-integrin) is a C-type lectin molecule binding mannose type carbohydrate pattern molecules thus activating phagocytosis (34). Despite lower CD209 expression GBM-DCs were functional in terms of effective FITC-dextran phagocytosis (Fig. 3). These phenotypic and functional analyses suggest that DC generation may also be performed from the peripheral blood of GBM patients, even if the DCs are generated from cells of the peripheral blood taken in the course of standard treatment.

Furthermore, we performed DC generation and antigenspecific $T$ cell assays in the presence of different concentrations 
of TMZ in vitro. In clinical application, the alkylating agent $\mathrm{TMZ}$ is given at a dose of $75 \mathrm{mg} / \mathrm{m}^{2} /$ day for 7 days per week during the time of irradiation therapy. After pausing the drug for four weeks, adjuvant therapy is performed with up to six cycles of $150-200 \mathrm{mg} / \mathrm{m}^{2} \mathrm{TMZ}$ for 5 days every 28 days (35).

TMZ serum levels of $5 \mu \mathrm{M}$ can be achieved in vivo after oral administration of the drug at a dose of $20 \mathrm{mg}$ per $\mathrm{m}^{2}$ body surface (4). Standard dosage of TMZ results in serum concentrations of up to $50 \mu \mathrm{M}$. We wondered whether TMZ at therapeutic concentrations might have a negative impact on the immunological functions of DCs and T cells, thus jeopardizing immunotherapy with DCs. Concentrations of TMZ used in our study covered the therapeutic range of TMZ serum concentrations 5-50 $\mu \mathrm{M}$ and beyond. No significant decrease of IL-12p70 secretion was observed within therapeutic levels (Fig. 5B). Nevertheless, we found that higher levels of TMZ indeed inhibit the generation of $\mathrm{CD} 83^{+} \mathrm{CD} 86^{+} \mathrm{DCs}$ and a concentration of $500 \mu \mathrm{M} \mathrm{TMZ}$ additionally decreased the number of viable DCs (Figs. 4 and 5A). Cytotoxic effects of TMZ could also be observed in other cell types as Kurzen et al (36) exposed endothelial cells to increasing concentrations of TMZ, with higher concentrations leading to significantly decreased cell numbers.

Moreover, T cells exposed to therapeutic doses of TMZ ranging from 5-50 $\mu \mathrm{M}$ during 7 days MLPC did not show impaired CMVpp65-specific $\mathrm{CD}^{+} \mathrm{T}$ cell reactivity and IFN- $\gamma$ secretion (Fig. 6). Chiba et al provided further evidence that the frequency of WT1-specific T cells from glioma patients remains stable, despite the lymphocytopenic effects of concomitant radiation/TMZ therapy in patients with newly diagnosed malignant glioma (37).

In summary, we demonstrated that $\mathrm{CD} 83^{+} \mathrm{CD} 86^{+}$ HLA-DRhi BDCA4hi CD209hi CD205 ${ }^{+}$CD $80^{+}$DCs can be generated from monocytes of GBM patients. Morphology and phenotype of DCs generated from GBM patients receiving TMZ therapy and at time of diagnosis showed no significant differences in comparison to healthy control persons. Most important, therapeutical doses of TMZ did not impair the function of either DCs or $\mathrm{CD}^{+} \mathrm{T}$ cells in vitro. These data strongly support the combination of TMZ chemotherapy with autologous DC immunotherapy as promising treatment option for glioblastoma.

\section{Acknowledgements}

We thank Ms. Goetz and Ms. Gross for excellent technical support. This research was supported by an internal grant of the faculty of medicine of the University of Rostock (Project number: 01/2010) and the German José Carreras Leukemia Foundation (DJCLS, project number: R09/14 and R10/03).

\section{References}

1. Van Meir EG, Hadjipanayis CG, Norden AD, Shu HK, Wen PY and Olson JJ: Exciting new advances in neuro-oncology: the avenue to a cure for malignant glioma. CA Cancer J Clin 60 166-193, 2010.

2. Stummer W, Reulen HJ, Meinel T, et al: ALA-Glioma Study Group. Extent of resection and survival in glioblastoma multiforme: identification of and adjustment for bias. Neurosurgery 62: $564-576,2008$
3. Stupp R, Hegi ME, Mason WP, et al: Effects of radiotherapy with concomitant and adjuvant temozolomide versus radiotherapy alone on survival in glioblastoma in a randomised phase III study: 5-year analysis of the EORTC-NCIC trial. Lancet Oncol 10: 459-466, 2009.

4. Agarwala SS and Kirkwood JM: Temozolomide. a novel alkylating agent with activity in the central nervous system, may improve the treatment of advanced metastatic melanoma. Oncologist 5: 144-151,2000.

5. Ardon H, Van Gool S, Lopes IS, et al: Integration of autologous dendritic cell-based immunotherapy in the primary treatment for patients with newly diagnosed glioblastoma multiforme: a pilot study. J Neurooncol 99: 261-272, 2010.

6. Ardon H, De Vleeschouwer S, Van Calenbergh F, et al: Adjuvant dendritic cell-based tumour vaccination for children with malignant brain tumours. Pediatr Blood Cancer 54: 519-525, 2010.

7. Van Gool S, Maes W, Ardon H, Verschuere T, Van Cauter S and De Vleeschouwer S: Dendritic cell therapy of high-grade gliomas. Brain Pathol 19: 694-712, 2009.

8. De Vleeschouwer S, Fieuws S, Rutkowski S, et al: Postoperative adjuvant dendritic cell-based immunotherapy in patients with relapsed glioblastoma multiforme. Clin Cancer Res 14: 3098-3104, 2008.

9. Liau LM, Prins RM, Kiertscher SM, et al: Dendritic cell vaccination in glioblastoma patients induces systemic and intracranial T-cell responses modulated by the local central nervous system tumor microenvironment. Clin Cancer Res 11: 55155525, 2005.

10. Pera MF, Köberle B and Masters JRW: Exceptional sensitivity of testicular germ cell tumour cell lines to the new anti-cancer agent, temozolomide. Br J Cancer 71: 904-905, 1995.

11. Li L, Schmitt A, Reinhardt P, et al: Reconstitution of CD40 and CD80 in dendritic cells generated from blasts of patients with acute myeloid leukemia. Cancer Immun 3: 8, 2003.

12. Li L, Reinhardt P, Schmitt A, et al: Dendritic cells generated from acute myeloid leukemia (AML) blasts maintain the expression of immunogenic leukemia associated antigens. Cancer Immunol Immunother 54: 685-693, 2005.

13. Li L, Giannopoulos K, Reinhardt P, et al: Immunotherapy for patients with acute myeloid leukemia using autologous dendritic cells generated from leukemic blasts. Int J Oncol 28: 855-861, 2006.

14. Schmitt A, Li L, Giannopoulos K, Greiner J, Reinhardt P, Wiesneth $M$ and Schmitt M: Quantitative expression of Toll-like receptor-2, -4 , and -9 in dendritic cells generated from blasts of patients with acute myeloid leukemia. Transfusion 48: 861-870, 2008.

15. Greiner J, Li L, Ringhoffer M, et al: Identification and characterization of epitopes of the receptor for hyaluronic acid-mediated motility (RHAMM/CD168) recognized by $\mathrm{CD}^{+} \mathrm{T}$ cells of HLA-A2-positive patients with acute myeloid leukemia. Blood 106: 938-945, 2005.

16. Schmitt M, Schmitt A, Rojewski MT, et al: RHAMM-R3 peptide vaccination in patients with acute myeloid leukemia, myelodysplastic syndrome, and multiple myeloma elicits immunologic and clinical responses. Blood 111: 1357-1365, 2008.

17. Schmitt M, Schmitt A, Reinhardt $\mathrm{P}$, et al: Opsonization with a trifunctional bispecific (alphaCD3 x alphaEpCAM) antibody results in efficient lysis in vitro and in vivo of EpCAM positive tumor cells by cytotoxic $\mathrm{T}$ lymphocytes. Int $\mathrm{J}$ Oncol 25: 841-848, 2004.

18. Shah MA and Schwartz GK: Cell cycle-mediated drug resistance: an emerging concept in cancer therapy. Clin Cancer Res 7: 2168-2181, 2001.

19. Liu G, Black KL and Yu JS: Sensitization of malignant glioma to chemotherapy through dendritic cell vaccination. Expert Rev Vaccines 5: 233-247, 2006.

20. Hatano M, Eguchi J, Tatsumi T, et al: EphA2 as a gliomaassociated antigen: a novel target for glioma vaccines. Neoplasia 7: 717-722, 2005

21. Schmitz M, Wehner R, Stevanovic S, et al: Identification of a naturally processed $\mathrm{T}$ cell epitope derived from the gliomaassociated protein SOX11. Cancer Lett 245: 331-336, 2007.

22. Liu G, Ying H, Zeng G, Wheeler CJ, Black KL and Yu JS: HER-2, gp100, and MAGE-1 are expressed in human glioblastoma and recognized by cytotoxic T cells. Cancer Res 64: 4980-4986, 2004.

23. Tang J, Flomenberg P, Harshyne L, Kenyon L and Andrews DW. Glioblastoma patients exhibit circulating tumor-specific CD8 ${ }^{+} \mathrm{T}$ cells. Clin Cancer Res 11: 5292-5299, 2005. 
24. Yu JS, Wheeler CJ, Zeltzer PM, et al: Vaccination of malignant glioma patients with peptide-pulsed dendritic cells elicits systemic cytotoxicity and intracranial T-cell infiltration. Cancer Res 61: 842-847, 2001.

25. Kobayashi T, Yamanaka R, Homma J, Tsuchiya N, Yajima N, Yoshida S and Tanaka R: Tumor mRNA-loaded dendritic cells elicit tumor-specific CD8(+) cytotoxic T cells in patients with malignant glioma. Cancer Immunol Immunother 52: 632-637, 2003.

26. Yamanaka R, Abe T, Yajima N, et al: Vaccination of recurrent glioma patients with tumour lysate-pulsed dendritic cells elicits immune responses: results of a clinical phase I/II trial. Br J Cancer 89: 1172-1179, 2003.

27. Caruso DA, Orme LM, Neale AM, et al: Results of a phase 1 study utilizing monocyte-derived dendritic cells pulsed with tumor RNA in children and young adults with brain cancer. Neuro Oncol 6: 236-246, 2004.

28. Yu JS, Liu G, Ying H, Yong WH, Black KL and Wheeler CJ: Vaccination with tumor lysate-pulsed dendritic cells elicits antigen-specific, cytotoxic T-cells in patients with malignant glioma. Cancer Res 64: 4973-4979, 2004.

29. Rutkowski S, De Vleeschouwer S, Kaempgen E, et al: Surgery and adjuvant dendritic cell-based tumour vaccination for patients with relapsed malignant glioma, a feasibility study. Br J Cancer 91: 1656-1662, 2004.

30. Maes W and Van Gool SW: Experimental immunotherapy for malignant glioma: lessons from two decades of research in the GL261 model. Cancer Immunol Immunother 60: 153-160, 2011.
31. Yamanaka R, Homma J, Yajima N, et al: Clinical evaluation of dendritic cell vaccination for patients with recurrent glioma: results of a clinical phase I/II trial. Clin Cancer Res 11: 4160-4167, 2005.

32. Tong AW, Papayoti MH, Netto G, Armstrong DT, Ordonez G, Lawson JM and Stone MJ: Growth-inhibitory effects of CD40 ligand (CD154) and its endogenous expression in human breast cancer. Clin Cancer Res 7: 691-703, 2001.

33. Shurin MR, Yurkovetsky ZR, Tourkova IL, Balkir L and Shurin GV: Inhibition of CD40 expression and CD40-mediated dendritic cell function by tumor-derived IL-10. Int J Cancer 101: 61-68, 2002.

34. McGreal E, Miller J and Gordon S: Ligand recognition by antigen-presenting cell C-type lectin receptors. Curr Opin Immunol 17: 18-24, 2005.

35. Stockhammer F, Misch M, Koch A, et al: Continuous low-dose temozolomide and celecoxib in recurrent glioblastoma. J Neurooncol 100: 407-415, 2010.

36. Kurzen H, Schmitt S, Näher H and Möhler T: Inhibition of angiogenesis by non-toxic doses of temozolomide. Anticancer Drugs 14: 515-522, 2003.

37. Chiba Y, Hashimoto N, Tsuboi A, et al: Effects of concomitant temozolomide and radiation therapies on WT1-specific T-cells in malignant glioma. Jpn J Clin Oncol 40: 395-403, 2010. 